\title{
Subsurface Monitoring using Low Frequency Wireless Signal Networks
}

\author{
Suk-Un Yoon, Zi Wang, Liang Cheng \\ Department of Computer Science and Engineering \\ Lehigh University \\ Bethlehem, PA, USA \\ \{suy309, ziw210\}@1ehigh.edu, cheng@cse.lehigh.edu
}

\author{
Ehsan Ghazanfari, Sibel Pamukcu, Muhannad T. Suleiman \\ Department of Civil and Environmental Engineering \\ Lehigh University \\ Bethlehem, PA, USA \\ \{ehg209, sp01, mts210\}@lehigh.edu
}

\begin{abstract}
The Wireless Signal Networks (WSNs) use the signal strength variation of wireless nodes in the host medium as the main indicator of events or physical property changes in the medium. With the concept and application of the Underground Wireless Signal Networks (UWSNs) where the host medium is a soil, the real-time global subsurface monitoring of wireless underground sensor networks can be achieved, whereas traditional wireless sensor networks provide point sensing and monitoring. In this paper, we present two representative experiments of subsurface monitoring such as leakage monitoring and earthquake monitoring using wireless sensor nodes. However, the deployment of underground wireless signal networks has more constraint than aboveground deployment, because the radio signal attenuation is much higher than above ground communication. To realize UWSNs for practical subsurface monitoring applications, it is necessary to extend the underground communication radius. In the paper, we provide the estimations of extending underground communication radius of underground wireless nodes based on the proposed underground radio propagation model [1]. Based on the estimations, we introduce a concept of Low Frequency Wireless Signal Networks (LFWSNs) for subsurface monitoring applications as well as an experimental platform for the low frequency wireless signal networks.
\end{abstract}

Index Terms-Wireless Signal Networks; Subsurface Monitoring; Low Frequency Wireless Signal Networks.

\section{INTRODUCTION}

Wireless sensor nodes can provide useful pervasive environmental information such as temperature, brightness, noise level, moisture, motion, air and water pollutants. The wireless sensor nodes can cooperatively monitor underground environments such as water and mineral content in the soils, oil leakage from oil reservoir, or land movement for landslide monitoring and earthquake monitoring. Traditional wireless sensors provide point sensing and monitoring where the sensing data are coming from sensor hardware that produce a measurable response to a physical property change. This paper introduces a new pervasive underground sensing method using the wireless signal from underground sensor nodes. The wireless signal strength information from networked sensors are used to produce a response to a underground condition change where the concept is called Wireless Signal Networks (WSNs). To provide practical pervasive underground sensing, we introduce Low Frequency Wireless Signal Networks (LFWSNs) which have better underground radio propagation.

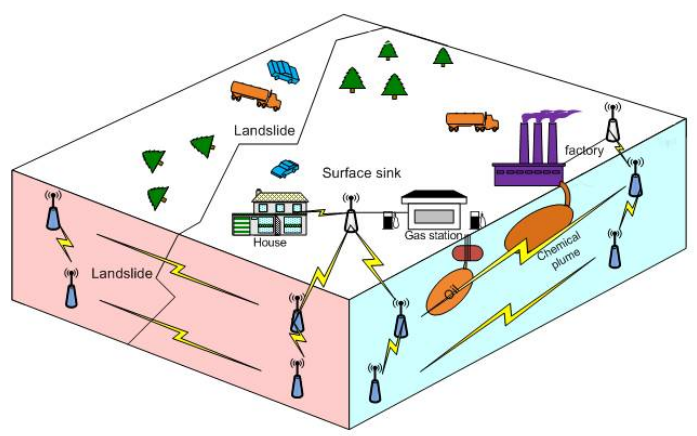

Fig. 1. Pervasive Subsurface Monitoring with Wireless Signal Networks

\section{A. Concept of Wireless Signal Networks}

The Wireless Signal Networks (WSNs) concept uses the electromagnetic (EM) signal strength variation as the main indicator of an event in the physical domain of the wireless signal network [2]. Soil properties such as density, water and mineral content are known to play important roles in determining losses of a propagating EM wave. When and if these physical properties of the host soil change during the evolution of a geo-event, they in turn affect the transmission quality and strength of the EM waves within the region of the event. Figure 1 shows the concept of pervasive subsurface monitoring with wireless signal networks for detection of landslide and chemical plume/oil leakage.

\section{B. Underground Radio Propagation}

In the air, the received signal strength is decaying with distance square, which is introduced by the Friis radio transmission formula. The received signal strength in soil is decaying much faster than air due the high attenuation of radio propagation in soil medium. An accurate and simple radio propagation model for underground low-power devices such as wireless sensor nodes is introduced and its performance is evaluated by real wireless sensor nodes (MICAz) [1]. The received signal strength in soils is defined as follows [1]:

$$
P_{r}(\rho)=K \frac{e^{-2 \alpha \rho}}{\rho^{2}}
$$

\section{Wireless Signal NeTWORKS}




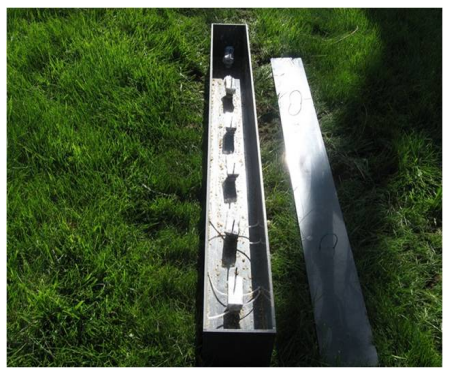

(a) A plastic box

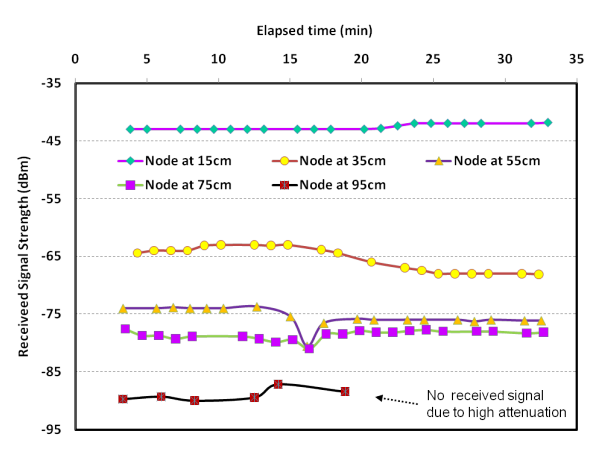

(b) Leakage monitoring

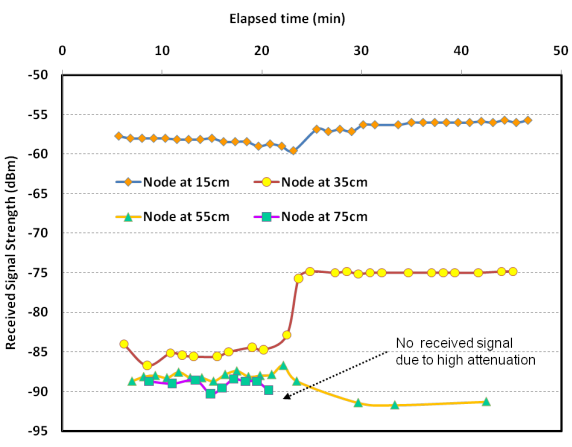

(c) Earthquake monitoring

Fig. 2. Applications of Wireless Signal Networks

where $\rho$ is the distance from the source; $K$ is a constant which includes transmit power, intrinsic wave impedance, and antenna gains; and $\alpha=\omega \sqrt{\frac{\mu \epsilon}{2}\left[\sqrt{1+\left(\frac{\sigma}{\omega \epsilon}\right)^{2}}-1\right]}$ is the attenuation constant of the medium, where $\omega$ is angular frequency, $\epsilon$ is the permittivity, $\mu$ is the permeability, and $\sigma$ is the electrical conductivity of the medium. Comparing the theoretical estimations of the underground radio propagation and the measured data using MICAz $(2.4 \mathrm{GHz})$, the theoretical model fits the measured data well within a $3.45 \mathrm{dBm}$ deviation or with an accuracy of $96.33 \%$ on average [1].

\section{Applications of Wireless Signal Networks}

To monitor underground environments, the underground radio propagation model can be used by underground wireless signal networks which use the wireless signal strength variation in the soils as the main indicator of an event. By analyzing the received signal strength, the wireless sensor networks can collect additional information from the wireless data carrier. The targeted events are subsurface hazards such as a landslide or earthquake which involve a lot of shifting and moving of earth masses, and the intrusion of a chemical plume which would dramatically change the physical properties of the host soil as shown in Figure 1. These events would affect the transmission of radio waves and the received signal strength in the region. Because the underground received signal strength deviation is very small, the subsurface events can be detected by the signal strength deviation as follows: $P_{r}(\rho, t+\Delta t) \geq \zeta \times P_{r}(\rho, t)$, where $\zeta$ is the deviation criterion which can be empirically determined based on the underground environments. If the sensor nodes are equipped with a moisture sensor or an accelerometer, the functional signal will provide more accurate detections such as the soil parameter variation and distance from the signal source. Using the underground radio propagation model in Equation 1, the distance from the source can be calculated by $\rho=W\left(\sqrt{\frac{\alpha^{2}}{K \times P_{r}}}\right) / \alpha$, where $W$ is the Lambert $W$ function.
Using the distance formulation underground sensor networks can detect subsurface events such as a landslide or earthquake by $\rho\left(P_{t}, t+\Delta t\right) \geq \zeta \times \rho\left(P_{t}, t\right)$, where $P_{t}$ is the transmit power.

To simulate these applications, two lab experiments were conducted: leakage monitoring and earthquake monitoring. As an underground medium, uniform size sandblast ( $D_{10}=0.40 \mathrm{~mm}, D_{30}=0.51 \mathrm{~mm}$, and $\left.D_{60}=0.62 \mathrm{~mm}\right)$ was used where $D_{x}$ is the diameter of the soil particles for which $x \%$ of the particles are finer. To control the soil properties, a plastic (PVC) box with dimensions $118 \times 13 \times 13 \mathrm{~cm}$ was made as shown in Figure 2(a) and the sensors were installed inside the box at every $15 \mathrm{~cm}$ or $20 \mathrm{~cm}$ from the signal transmitter. In each experiment, the geo-parameters (water content, salinity, compaction, and soil gradation) and physical properties of the environment such as temperature which affects the electric conductivity and permittivity of the medium were controlled.

1) Leakage monitoring: The experiment was conducted in wet sand with $12 \%$ water content for 35 minutes. 15 minutes after from the beginning of the test, a water leakage event was simulated around one of the nodes located at $55 \mathrm{~cm}$ from the sender. After the water leakage event, the receivers located behind $55 \mathrm{~cm}$ from the sender showed a lower received power due to more attenuation caused by the increase in water content as shown in Figure 2(b). The node located at $95 \mathrm{~cm}$ lost the signal from the source due to high attenuation and no data was recorded. With the decreased received signal information, both the time of the occurrence and location of the event could be detected.

2) Earthquake monitoring: In this experiment, four sensor nodes were deployed and simulated earthquakes occurred after 22 minutes from the start of the test. The earthquake simulation was made by shaking and vibrating the box on all sides. During the earthquake event, the first two nodes moved close to the sender and the last two nodes moved away from the sender. After the earthquake event, the received signal strengths of the nodes located at $15 \mathrm{~cm}$ and $35 \mathrm{~cm}$ increased due to their decreased distance from the sender while the received 


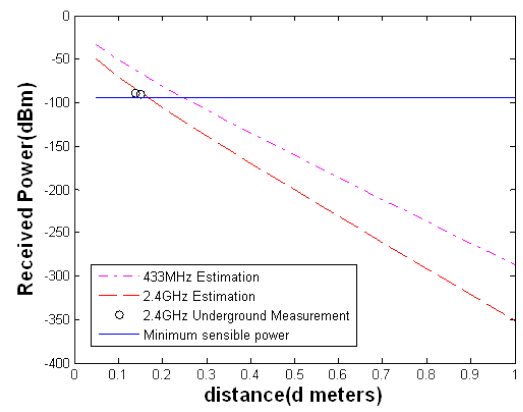

(a) With $2.4 \mathrm{GHz}$ and $433 \mathrm{MHz}$

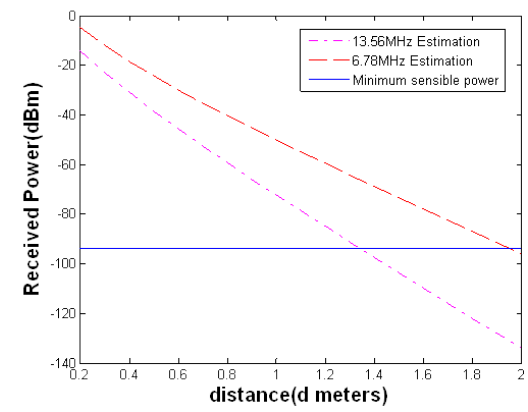

(b) With $13.56 \mathrm{MHz}$ and $6.78 \mathrm{MHz}$

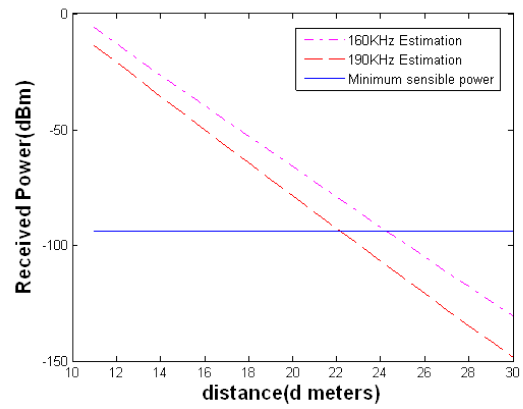

(c) With $160 \mathrm{KHz}$ and $190 \mathrm{KHz}$

Fig. 3. Underground Radio Signal Attenuation

signal strength of the node located at $55 \mathrm{~cm}$ decreased due to its increased distance from the sender as shown in Figure 2(c). The node located at $75 \mathrm{~cm}$ lost the signal from the source after the earthquake and no data was recorded. As seen from this simulation experiment, there is a potential to detect and monitor earthquake events using information from the received signal strength.

\section{Low FreQuency Wireless Signal NeTworks}

\section{A. Challenges of Wireless Signal Networks}

The deployment of sensor networks in challenging environments such as underground incurs new problems as following:

1) Installation and Management: The installation and management of underground sensors are much more difficult than aboveground networks because digging a hole and finding sensor underground are not easy tasks. Even the sensor position is easily traceable; the sensor can be damaged when digging. Thus, before deployment underground sensor networks, the network and topology should be designed to minimize installation and management costs. For example, the sensor in high energy consumption can be deployed in shallow depth which will be easier to manage.

2) Battery Power: Replacing battery of underground sensors is quite difficult due to their locations. To avoid replacing battery, high capacity battery and power saving sensor operation such as sleep mode can be used. If sensor nodes adopt high capacity battery such as D batteries $(15000 \mathrm{mAh})$ instead of widely used AA batteries $(2000 \sim 2400 \mathrm{mAh})$, the sensor nodes' lifetime can be extended about 7 times longer. For the sake of minimizing energy consumption of underground sensors operation, sleep mode and long data reporting interval can be adopted.

3) Communication Radius: The communication radius in soil is much shorter than air due the high attenuation of radio propagation in soil medium. Based on the underground radio propagation model and the field measurements, the communication radius of the commercial wireless sensors such as $2.4 \mathrm{GHz} \mathrm{MICAz}$ and $433 \mathrm{MHz}$ MICA2 are about $20 \mathrm{~cm}$ and
$30 \mathrm{~cm}$ in wet clay type soil (measured electrical conductivity = $78 \mathrm{mS}$, estimated relative permittivity $=30$ ) sampled in Lehigh University Goodman Campus as shown in Figure 3(a).

\section{B. Estimation of Underground Radio Propagation}

To overcome the high attenuation and extend communication radius in soil, three parameters can be controlled such as using high transmission power, using high gain antenna, and using low radio frequency. In case of high transmission power and high gain antenna, the benefit of extending communication radius is not enough to design practical underground applications. If the underground sensors use a low frequency, the communication radius can be extended more efficiently. There are candidates of low frequency bands for underground communication such as LowFER (Low-Frequency Experimental Radio) of 160 190 KHz in the United States and Canada, and low frequency ISM (industrial, scientific and medical) bands of $6.78 \mathrm{MHz}$ and $13.56 \mathrm{MHz}$. ISM bands are radio bands reserved internationally for the use of radio frequency for industrial, scientific and medical purposes. LowFER is a license-free form of two-way radio communications practiced on frequencies below $500 \mathrm{KHz}$ [3]. In the United States, one permits the use of up to 1 Watt of power and a 15 meter long antenna between $160 \sim 190 \mathrm{KHz}$ with no license requirement [4]. We can achieve $1.3 \sim 2 \mathrm{~m}$ communication with $6.78 \mathrm{MHz}$ and $13.56 \mathrm{MHz}$ ISM bands, and $20 \sim 25 \mathrm{~m}$ communication with $160 \sim 190 \mathrm{KHz}$ LowFER bands even in wet clay underground with 1 Watt Tx power and the same MICA's antenna gain as shown in Figure 3(b) and 3(c). In the future, the performances (e.g., received signal strength versus distance) of low frequency communications with real systems and low frequency antennas will be evaluated and compared with the theoretical analysis. We currently configured two USRP nodes operating in low frequencies such as $190 \mathrm{KHz}, 6.78 \mathrm{MHz}$, and $13.56 \mathrm{MHz}$ with two daughter boards (BasicTX/RX and LFTX/RX) and Tri-band 7-inch omnidirectional vertical antenna (VERT400 antenna [5]). We will customize commercial low frequency antenna for subsurface monitoring applications 


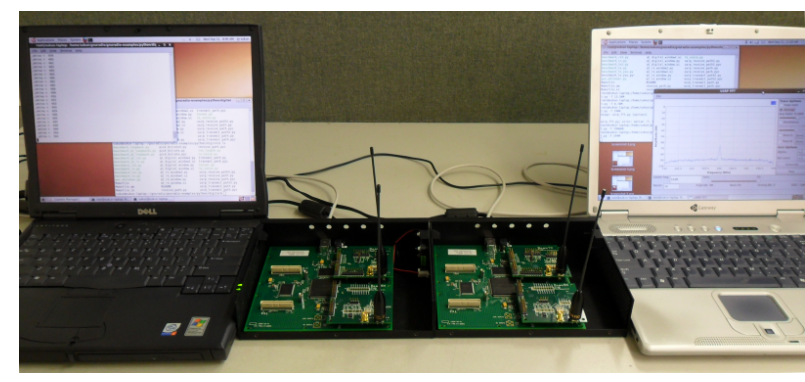

Fig. 4. USRP Setup for Low Frequency Communications

and evaluate the performance of the system. To enhance the low frequency subsurface communication, we will design experimental antennas which are compact and suitable for subsurface installation and compare their performance with commercial one.

\section{An Experimental Low Frequency Platform using USRP}

USRP (Universal Software Radio Peripheral) is an experimental platform of a GNU radio or software-defined radio (SDR) system where components that have been typically implemented in hardware (e.g. mixers, filters, amplifiers, modulators/demodulators, detectors, etc.) are instead implemented by means of software on a personal computer or embedded computing devices [6]. We use USRP boards and two daughter boards for the evaluation platform of low frequency operation via radio reconfiguration. The BasicTX and BasicRX boards are designed for use with external RF frontends operating from 1 to $250 \mathrm{MHz}$ frequency bands [5]. LFTX and LFRX are similar to the BasicTX and BasicRX, but the operating frequency bands are from $\mathrm{DC}$ to $30 \mathrm{MHz}$ with differential amplifiers and low pass filters [5]. We verified the USRP system can work on the low frequency bands which are suitable for underground communications. Using two USRP mother boards equipped with BasicTX/RX and LFTX/RX daughter boards, the communication systems working on low frequency bands are established as shown in Figure 4. We tested three candidates of low frequency bands such as $13.56 \mathrm{MHz}$ ISM band, $6.78 \mathrm{MHz}$ ISM band, and $190 \mathrm{KHz}$ LowFER band. In the experiments, one host PC (Linux OS) is sending data while another host PC is receiving the date. Instead of printing received data, the receiver can run spectrum analyzer to show the RF signal level. The receiver shows increased signal level when it is receiving data.

\section{Low Frequency Sensor Hardware Design}

To realize low frequency underground sensor networks, we designed a new hardware platform which is called Reconfigurable Radio Sensor Networks (RRSNs) based on the MICA2/MICAz and USRP platforms. The main processing parts (i.e., CPU, memory, etc.) of the reconfigurable radio sensor are coming from MICA2/MICAz and the reconfigurable radio parts for the low frequency operation are coming from USRP system. The significant difference of reconfigurable

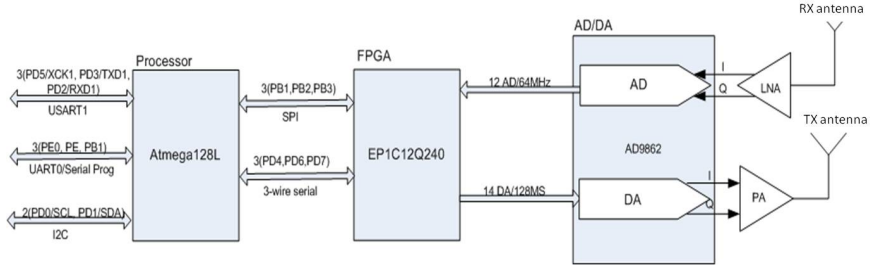

Fig. 5. The block diagram of reconfigurable radio sensor node

radio low frequency sensor from USRP is the implementation of radio communication system. Because the sensor node has low computational CPU, it cannot implement the communication system in softwares. In the reconfigurable radio sensor networks, the traditional radio communication blocks (e.g. mixers, filters, amplifiers, modulators/demodulators, detectors, etc.) are implemented in FPGA (Field Programmable Gate Array). The reconfigurable radio sensor node can have multiple radio communication blocks working on different frequency bands ranging from $\mathrm{KHz}$ to $\mathrm{GHz}$. Depending on the requirements of frequency ranges, the sensor node can be equipped with multiple communications blocks and antennas. The main block diagram of reconfigurable radio sensor node is shown in Figure 5. We finished the PCB schematic design and produced PCB boards to develop the reconfigurable sensor node. The FPGA programming for low frequency reconfigurable radio sensor platform is in progress.

\section{CONCLUSION}

The paper proposes a new concept of Low Frequency Wireless Signal Networks (LFWSNs) with theoretical estimations and practical experimental solutions. The paper also presents the design and development in progress of reconfigurable radio sensor networks for low frequency operations. In the future, we will customize antennas in low frequency bands to extend underground communication distance, and evaluate the performances of low frequency communications with USRP system and the designed reconfigurable radio sensor networks.

\section{ACKNOWLEDGMENT}

This material is based upon work partly supported by the National Science Foundation (NSF) under Grant No. 0855603. Any opinions, findings, and conclusions or recommendations expressed in this material are those of the author(s) and do not necessarily reflect the views of the NSF.

\section{REFERENCES}

[1] Suk-Un Yoon, Liang Cheng, Ehsan Ghazanfari, Sibel Pamukcu, and Muhannad T. Suleiman, A Radio Propagation Model for Wireless Underground Sensor Networks, IEEE Globecom, 2011.

[2] Xu Li, Tae sup Yun, Liang Cheng, Sibel Pamukcu, and Yi Dong, Insitu Geo-characterization Using Wireless Functional Signals, SPIE Smart Structures/NDE 2010, San Diego, California, March 7-11 2010.

[3] http://en.wikipedia.org/wiki/LowFER

[4] http://www.lwca.org/sitepage/part15/whatisit.htm

[5] http://www.ettus.com/

[6] Markus Dillinger, Kambiz Madani, Nancy Alonistioti, Software Defined Radio: Architectures, Systems and Functions, Wiley \& Sons, 2003, ISBN 0470851643 . 